\title{
Design and Additive Manufacturing of Lattice-based Cellular Solids at Building Scale
}

\author{
Roberto Naboni \\ ACTLAB - Politecnico di Milano, ABC Department, \\ Italy \\ roberto.naboni@polimi.it \\ Anja Kunic \\ ACTLAB - Politecnico di Milano, Italy \\ anja.kunic@gmail.com
}

\begin{abstract}
The amounts of material that is being extracted, harvested and consumed in the last decades is increasing tremendously and bringing to the serious problem of resource scarcity. As a direct consequence to this claim, designers are challenged to rethink architecture and develop new ways of confronting with materials. A potential answer to this problem is the exploration of computational logics for architectural design and fabrication inspired by the observation of biological formations. This work explores how the biological model of bone microstructure can be applied to a larger scale architecture that is structurally responsive, by means of computational design and Additive Manufacturing.
\end{abstract}

Keywords: Functionally Graded Trabecular Tectonics, Digital fabrication, Additive Manufacturing, Computational Design, Biomimetics

\section{Introduction}

\section{Scarcity of resources}

Overconsumption of resources is one of the greatest challenges of 21 st century. The tremendous increase in the amounts of material that is being extracted, harvested and consumed in the last decades is bringing to the serious problem of resource scarcity (Fig. 1). Coal, oil and gas are not only becoming increasingly scarce, but are also accelerating emergence of sensitive global crisis leading to climate change and exceeded $\mathrm{CO} 2$ emissions.

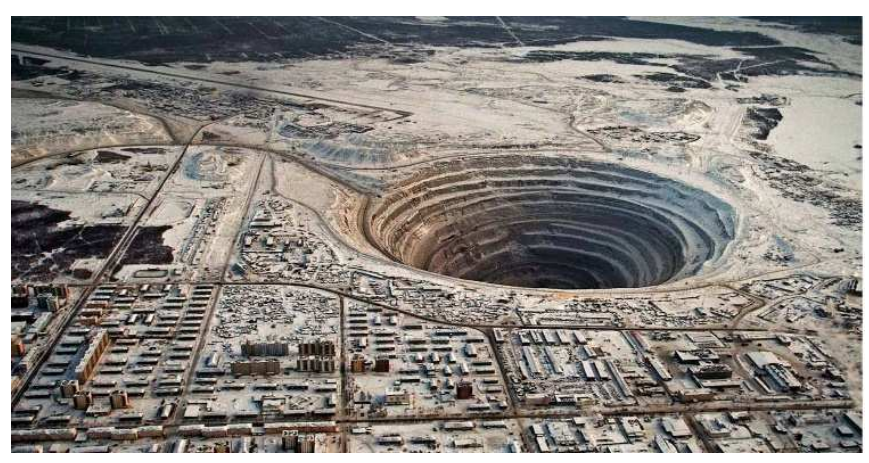

Figure 1: Mirny diamond mine in Eastern Siberia, Russia

Even though the most usual associations to the pollution and $\mathrm{CO} 2$ emission are cars and aeroplanes, these are not the crucial culprits. In fact, another area that is more critical and far more problematic is the building construction, which causes, directly or indirectly, $80 \%$ of $\mathrm{CO} 2$ emissions. Therefore, the architects more than any other responsible segments of society and professional groups are challenged to rethink the way of designing, building and confronting with materials. They will have necessarily to adapt to the post-fossil age and make a crucial contribution to the ecological turnaround through the intelligent and looking-forward design, use of materials, technologies, recycling requirements and energy use.

\section{Improving building resilience by learning from biological models}

In this paper the main focus is brought to the investigation of cellular solids as a tectonic system in architecture that is based on a complex form articulation to conveniently compensate material scarcity. In particular, the hierarchical structure of bones is considered as the most prominent example of lightweight and at the same time structurally efficient natural systems. This work explores how the biological model of bone microstructure can be applied to a larger scale architectural prototypes, by means of computational design and Additive Manufacturing (AM).

Learning from nature and biological formations has become a meaningful and crucial approach for contemporary architects, especially for those who foster a future that does not compete with nature but coexists with it. As a response to the natural disasters worldwide, especially those geologically associated such as tsunami and earthquakes, which have proven their destructive power over the current built environment, architects and structural engineers have found in biomimicry an ecological approach in order to improve future building's disaster resilience.

In his dissertation Bio-Structural Analogues in Architecture, Joseph Lim (2009) explains that 'the central to the development of architectural concept and design strategy is a form of technological thinking which drew inspiration from other forms of knowledge'. Scientific insights in the technological solutions promoted by nature and their links to 
architecture has led to a bottom up approach for more resilient structural design. As D'Arcy W. Thompson (1945) wrote, every form in Nature is essentially a product of a set of forces acting on it. This technological feature of the living structures is a parameter that defines the level of resilience of their morphology, since their tessellation grows in intrinsic relationship with the ecosystem and the natural flows within it.

\section{Cellular solids in nature}

When modern man builds large load-bearing structures, he uses dense solids: steel, concrete, glass. When nature does the same, she generally uses cellular materials: wood, bone, coral. There must be a good reason for it (Ashby 2000).

Cells are fundamental forms of life, the basic construction units of natural organisms which have been explored diffusely since their discovery by Robert Hooke, described in the seminal work Micrographia (Hooke, 1665). In the field of architecture, constructions based on assembly of cells with solid edges or faces packed together to fill a space are called cellular solids (Gibson and Ashby, 1997). Due to their inherent mechanical properties such as high strength to weight ratio, structural stiffness, energy absorption and other thermal, acoustic and electrical properties, cellular solids have become highly significant and attractive to many designers and scholars (Gibson, 2005). Examples of such structures are widely spread in nature (Flg. 2) and they can be found in various structural configurations. The different level of connectivity of cellular structures (topology) is the main parameter that influences the behaviour of the material/structure, together with its relative density $(\rho)$. The last one is defined as the ratio between the overall density of the cellular material $(\rho)$ divided by the one of the solid from which the cell walls are made $\left(\rho_{s}\right)$. When cell walls thicken and the pore space shrinks, the relative density increases. By definition cellular materials have a relative density inferior to 0.3 (Gibson and Ashby, 1997).

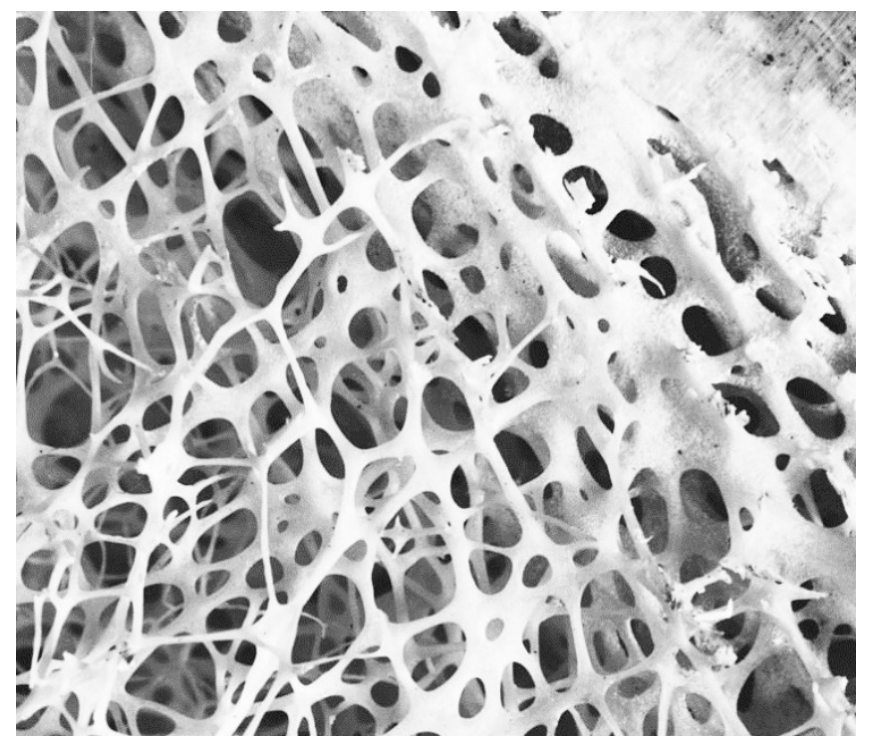

Figure 2: Type of of cellular structures found in nature: Open cell structure in cancellous bone

In this paper, an experimental skin system based on cellular solids is implemented through the combined use of advanced computational design tools and Additive Manufacturing. In particular, the study focuses on the investigation of lattice structures, a model that has an efficient way of structuring material (Gibson 2005) that are based on the open-cell configurations. Fundamentally, cellular lattice structures are composed of an interconnected network of struts, pin-jointed or rigidly bonded at their connections (Ashby, 2005). At one level, they can be analyzed using classical methods of mechanics such space frames, while on the other side, within a certain scale range, lattice can be considered as a material, with its own set of effective properties, allowing direct comparison with homogeneous materials.

Among the examples of lattice cellular solids in nature, the hierarchical structure of bones is considered as one of the most prominent lightweight and structurally efficient natural systems. Bones are made of a composite material that is about 95\% hard calcium-based mineral (hydroxyapatite) marbled with an elastic protein (collagen). The cortical bone makes up the exterior of the bone, while cancellous bone is found in the interior. This has high material efficiency because of its constitutional microstructure based on cells named trabeculae, that are formed through an iterative load-responsive process. Here, an emergent latticework of fibers constitutes a cellular microstructure informed by its loading conditions, which varies in porosity, and in orientation to align with the main stress trajectories to withstand both tensile and compressive forces (Benyus, 2002) (Fig. 3).

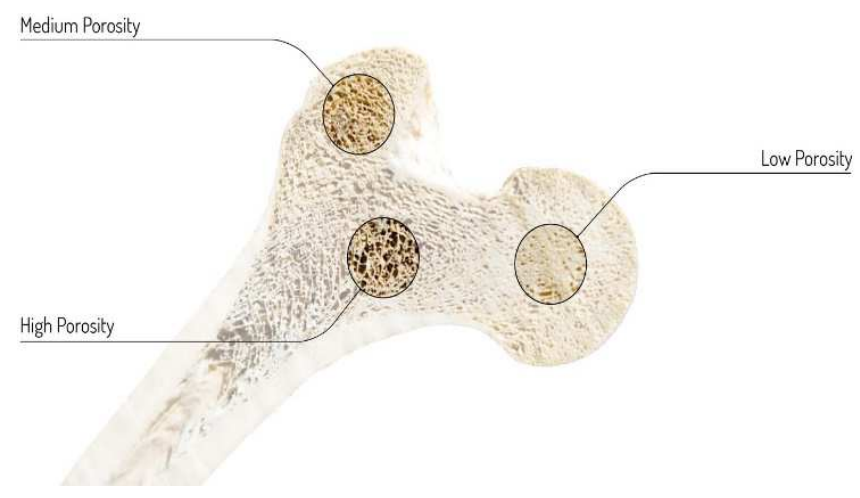

Figure 3: Section of a human femur bone showing degrees of porosity according to a load-responsive material organization

Interestingly, the process of bone remodeling is responsive to variable loading conditions which an individual can encounter during our life. In particular, this process is subjected to the simultaneous action of two cells - osteoblasts and osteoclasts, that are evaluating local strain values within the bone trabecular structure and adding or removing material accordingly. High strain levels indicate that the bone is weaker than expected and osteoblasts will compensate by adding material in order to reduce strain. Analogously, excessively low strain levels show an unneeded over-mineralization, and the need for osteoclasts to remove material. The balance between these two processes therefore provides a converging point where function and structure are optimized (Turner, 2012). This specific formation process can be synthesized in an algorithm which constitutes the procedural base for the generation of the load-responsive cellular envelope tackled in this paper (Fig. 4). 


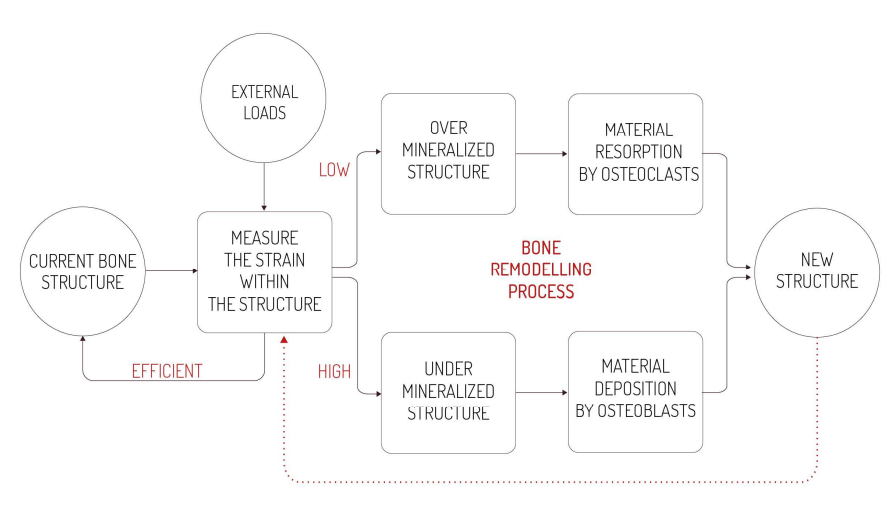

Figure 4: Algorithmic interpretation of the bone remodeling process (adapted from J.S. Turner, 2012)

\section{Methodological Procedures Computational workflow}

Specific logics pertaining to the bone remodelling process, such as the informed variation of porosity/relative density and orientation are here developed into a computational workflow for the design, optimization and fabrication of the so called Functionally Graded Trabecular Tectonics, an innovative system for building skins (Fig. 5). The main aim of this method is not to focus on mimicking the forms of nature, but rather to implement the main logics and technological solutions of biomimetic formation processes. As in nature, the main goal is to provide a general methodology of sustainable materialization that is to be applied to a potentially infinite variety of formal and loading conditions. This involves the elaboration of a customized algorithmic workflows which are generating and materializing lattice structures informed by specific mechanical behaviour. Analogous to the bone remodelling process, this workflow proposes a method which performs an iterative macroscale mechanical analysis with Finite Element Methods (FEM) to compute a very specific material organization or topology optimization of free-form building envelopes. The outcome of this analysis is then directly translated into a lattice microstructure which, in common with the trabecular bone, varies in porosity according to local stress values, and in size to best fit specific shape features. In this process, main input parameters are material properties and fabrication constraints of AM, overall geometry and boundary conditions. Variations in any of these would generate different outputs. Morphological, material and performance information is read, analyzed and modified iteratively.

A particular advantage of this approach is the possibility to be applied to a wide range of materials. Surely, mechanical properties of cellular solids are governed by those of the solid material from which they are made of, yet the most influencing is their topology and relative density. In particular: form does matter, more than material. For this reason, the investigation focuses on the use of Fused Deposition Modeling (FDM) with thermos-polymers - an additive method that ensure high complexity and control, relative geometrical freedom, and the use of weight and cost effective materials. This naturally takes advantage from the fact that thermoplastic polymers can be modeled into any desired shape while almost entirely preserving mechanical and thermal properties. The focus is questioning and proving the viability of FDM within the context of a laboratory prototype.

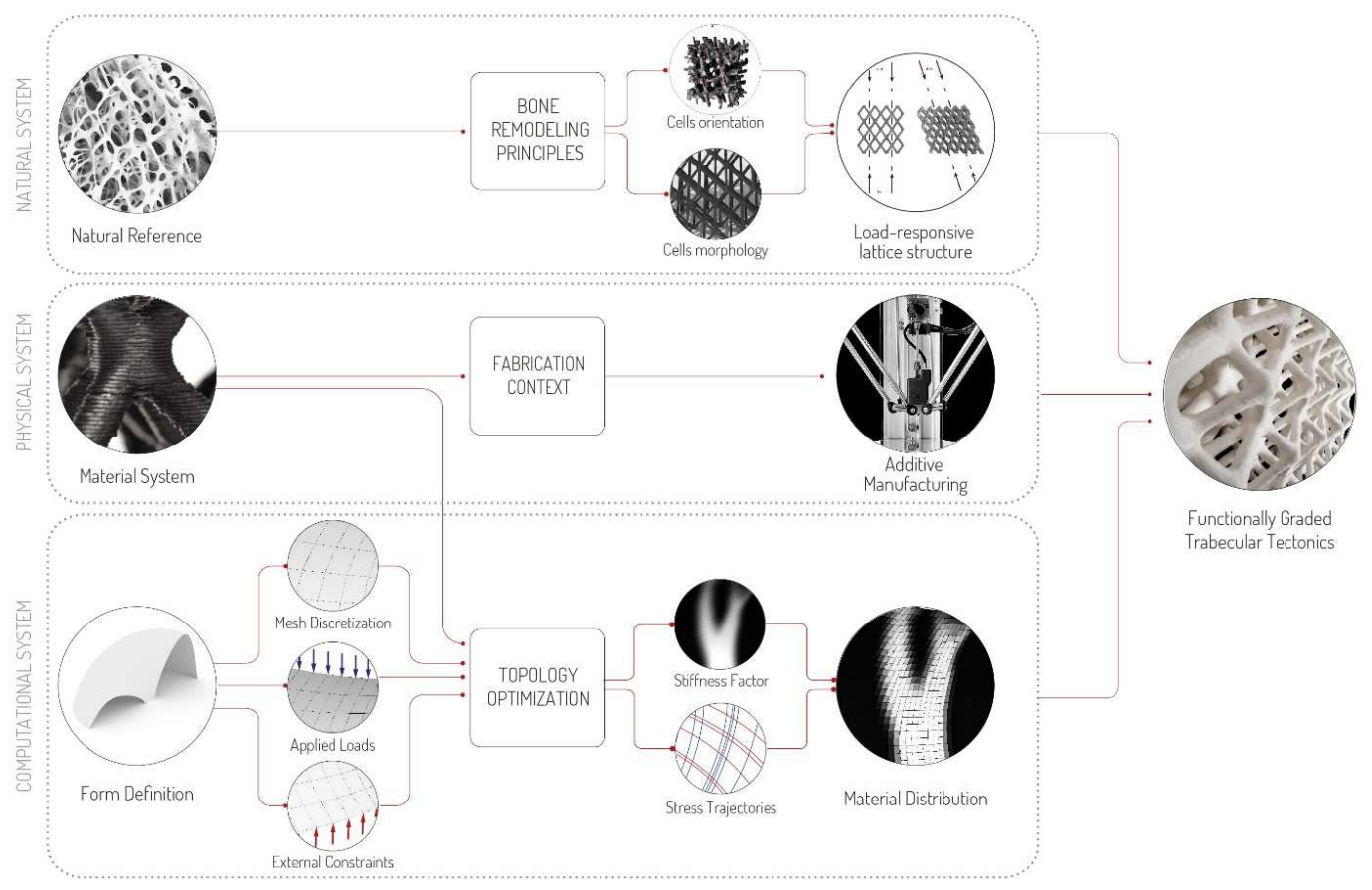

Figure 5: Scheme of the overall design workflow, involving computational tools, fabrication procedures and material information 


\section{Material system of additively manufactured thermos-polymers}

While natural polymers such as wool, cotton, silk, wood and leather have been known and used since ancient times, the synthetic polymers - the man made polymers, known as plastics, started to emerge in the early twentieth century. However, due to chemists' ability to engineer them to yield any desired set of properties (strength, stiffness, density, heat resistance, electrical conductivity), synthetic polymers have greatly and rapidly expanded in their role within the modern industrial economy. Design options that these new materials offered were quickly discovered and so it was not long before they took part in everyday building practice. Shapes that had been impossible in the past, were now added to vocabulary of industry and design (Knippers et al. 2010).

Thermo-Polymers or thermo softening plastics, consist of linear or branching molecular chains that do not form chemical bonds with each others. Instead, they are held together by weak physical forces or the so called secondary valency forces that are broken down when the material is heated. This allows the molecular chain to move which means that polymer material becomes soft and mouldable (Knippers et al. 2010). Due to their various mechanical, physical and thermal properties, as well as the ability to be manufactured in any desired shape and configuration, thermo polymers have taken a significant role in filament production for Additive Manufacturing (Fig. 6) and in that way opened new scenarios for their application in architecture, product design and manufacturing industry in general.

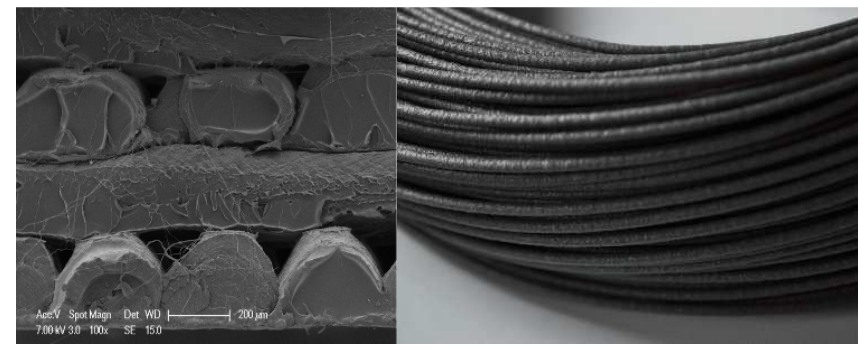

Figure 6: The images are showing two different material compositions of FDM thermo polymers. Left: microscopic view of 3d printed layers of PLA (Poly lactic acid); Right: PLA filament for FDM

Within the field of constructions, the shift from prototyping to direct manufacturing is mainly connected to material improvement, which in comparison with product design is more complicated to achieve. Material characteristics and behaviour, mechanical properties and dimensional requirements are key elements in evaluating the use of $\mathrm{AM}$ for large scale applications (Naboni \& Paoletti, 2015). Therefore, the exploration of a material system should be held in order to understand the way it can be exploited, with a rigorous multiscalar analysis of the material coupled with the fabrication system that will be used (Hensel, 2011). This process starts with analyzing the materialization process through fabrication experiments and the observation of their geometrical and mechanical characteristics (Fig. 7). As result, a set of specific boundary conditions for the fabrication systems, involving machinic, software and material interdependencies is defined.
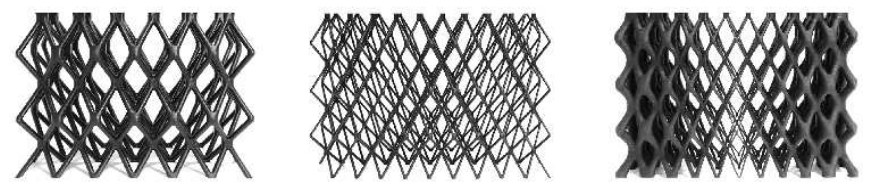

Figure 7: Experimental components showing different microstructure configurations with variable relative density

In the frame of this research it is used a delta-robot, a typology of printer intrinsically agile that guarantees an ideal travel speed for the production of discontinuous geometries such as the lattice structures (Fig. 8). The employed material is High Performance, biodegradable PLA (Polylactic Acid), a polymer with discrete mechanical properties which are leveraged by its superior printability. An extensive campaign of fabrication tests have been conducted with it to define print settings in relation to geometric constraints, printing time, printing resolution and mechanical resistance of the lattice microstructure. Among various aspects, an important one emerged in the necessity of evaluating models to be printed according to geometry limitations in overhanging angles, to avoid the need of support geometries with consequent inefficiency in the use of material. The relation between the deviation angle from the vertical axis and the number and thickness of shell elements is fundamentally driving the resolution and refinement of the production.

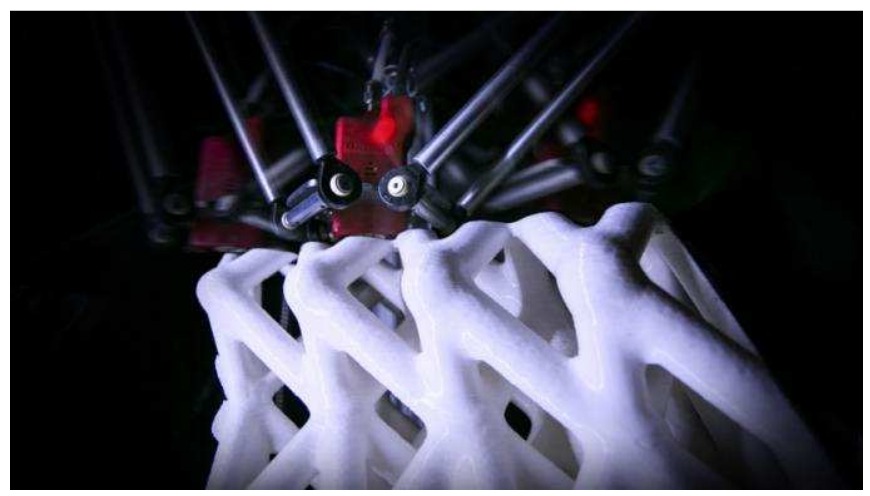

Figure 8: Printing process of the lattice structure by Delta Wasp robot

\section{Study on lattice cell typologies}

The definition of the base unit cell plays the main role in development of a cellular solid structure. In nature this is direct expression of a material system, which accommodates the biological and mechanical needs of an organism. This implies that the above-mentioned geometry constraints of FDM are to be taken first into account in the critical evaluation. A comparative multicriteria analysis of typical three dimensional cells have been conducted, with an evaluation of printability, relative density and visual permeability. Eight typologies have been analyzed: orthogonal grid $(A)$, star $(B)$, tesseract $(C)$, octahedron (D), cross $(E)$, octet $(F)$, vintiles $(G)$ and diamond (H) (Fig. 9). Each specimen is bounded in a $10000 \mathrm{~mm}^{3}$ cube and all the struts have a sectional diameter equal to $10 \mathrm{~mm}$. 
An analysis of the geometry constraints has been carried out, focusing on the evaluation of overhanging angles. Considering the $X Y$ plane as the leaning plane, a critical threshold for printability is set at $65^{\circ}$ angle deviation from the vertical axis. Printing angles below this value guarantees production speed and quality, whereas larger angles can be problematic, in particular with thicker layer heights, as emerged in the description of the material system. From the cell analysis, the octahedron (D) and diamond (E) cells show optimal features for this fabrication process.

In the analysis of relative density are highlighted large differences: on one hand, the Octahedron and Diamond have the lowest relative density of 0.18 and 0.10 . On the other hand, cells such as the Octet and the Tesseract have the highest relative density over 0.50 , meaning that more than half of the bounding box is occupied by the cell struts, resulting in a stiffer but heavier structure.

Finally, visual permeability is measured in respect to the projection of the unit cells on a vertical plane using a $30^{\circ}$ angle of view. This analysis highlights again strong differences among the samples, being the dimensions of the projected areas ranging from $4900 \mathrm{~mm}^{2}$ to $12100 \mathrm{~mm}^{2}$. Considering that the projected area of the bounding box is $16600 \mathrm{~mm}^{2}$, the octahedron with its area of $4900 \mathrm{~mm}^{2}$ obstructs about $1 / 3$ of the visual field, while the octet cell blocks around $3 / 4$ of the view with a projected area of $12100 \mathrm{~mm}^{2}$. Everything examined, octahedral cells have proven to be ideal to guarantee a streamlined production while offering a degree of freedom allowing variable mechanical and visual features.

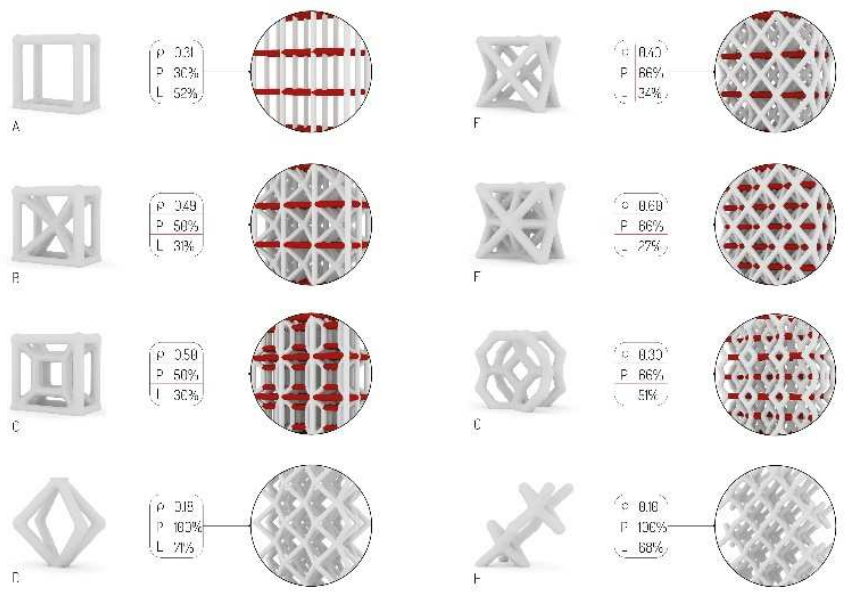

Figure 9: The image shows eight different unit cells typologies for the Cellular Lattice Structure and their observed characteristics; first column shows unit cell types: A - orthogonal grid, B - star, C tesseract, D - octahedron, E - cross, F - octet, G - vintiles and H diamond; second column shows the relative density $(\rho)$, printability

$(P)$ and light permeability $(L)$; third column shows the repeated unit cell in a skin system, highlighting in red elements that are not possible to be fabricated with FDM

\section{Design and fabrication of Functionally Graded Trabecular Tectonics}

As previously explained, the main characteristic of the Functionally Graded Trabecular Tectonic system is the ability of the microstructural configuration to adapt to various loading conditions and so to obtain the most optimized solution for structural resilience. Similarly to the trabecular bone, the lattice microstructure is formed in the response to the stresses acting on it, and therefore it is defined as a load-responsive structure. The overall process is fully developed within the computational environment, through the set of algorithms dealing with geometrical, structural and fabrication constraints, where the optimal solution is defined, compromising all the involved parameters.

Through the use of Topology Optimization (TO) principles, the structural performance and resilience can be anticipated for different situations of mechanical changes and loading parameters. Topology Optimization (TO) is often used as an early-stage design tool to give the designer an insight into an efficient structural layout. However, in this work the output of this analysis is used to directly inform the generation of a continuous lattice structure.

The TO is fed with two-dimensional free-form shapes, which represent a "draft" of building envelope configurations to be evaluated, along with a description of specific boundary conditions such as loads, constraints and material properties. An algorithm based on the Solid Isotropic Material Penalization (SIMP) iteratively computes stiffness values and allocates material in a multi-phase process which tends to converge to $0 / 1$ values. These are zones with lowest/highest density of material, respectively represented with an interpolation of black/white color values (Bends 酶 e and Sigmund, 2003). This representation is then converted into a Functionally Graded Trabecular Tectonics, where mechanical behaviours provide the needed information to evolve a base polyhedron into highly specific cells with locally optimized cell dimensions and orientation, struts diameter and section as well as material characteristics. The algorithmic workflow generates a grayscale representation of the desired stiffness values, which informs the sizing of each single strut diameter (Fig.10).

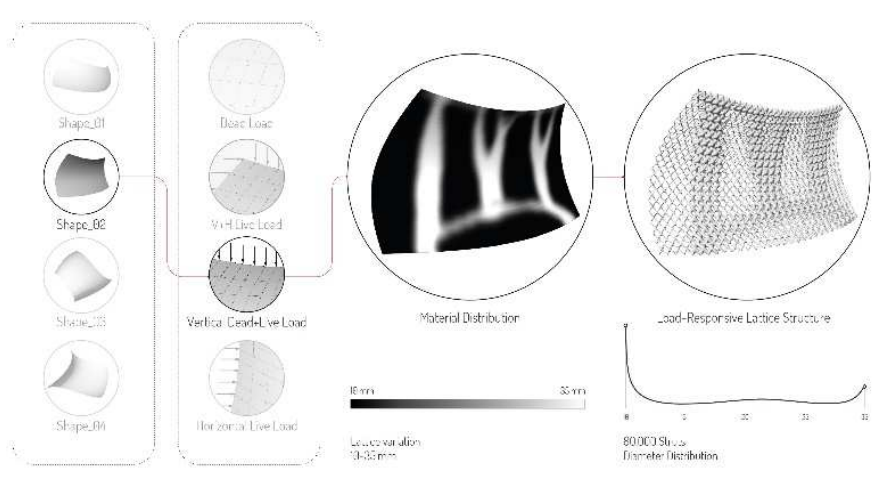

Figure 10: Workflow of the lattice cellular structure generation from the initial shape definition

Finally, building upon the previously developed research on additively manufactured building envelopes (Naboni et, al. 2016) and following the overall process and methodology including topology optimization, structural configuration and fabrication experiments, a full scale mock-up has been prototyped to address construction and fabrication aspects of the load bearing structure (Fig. 11). Starting from the selection of a free-form shape, this is evaluated under different external 
loading conditions, added to its self-weight, obtaining different patterns of material distribution until the convergence to the optimal one.

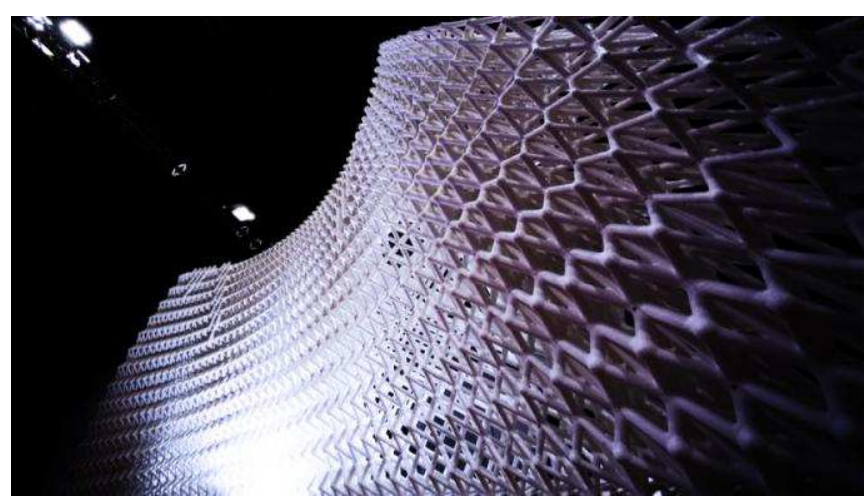

Figure 11: Full scale mock-up of a 3D printed Load-Responsive Lattice Structure

\section{Results}

The research develops a system for free-form building envelopes based on load-responsive lattice structures. The design process is inspired by the formation of cellular bone microstructure, in particular in the creation of gradients of porosity and cell orientation to improve the response to variable loading conditions and foster structural resilience. A novel algorithm-based workflow for the design of complex building skins is introduced. This is articulated in: an iterative procedure for FEM analysis of complex envelope shapes, which suggests optimal material distribution and a process of analysis of FDM material system to define base unit cell for the cellular system. Lattice samples at various scales are successfully prototyped and tested towards the validation of their application in construction. A final full scale mock-up is produced as a proof-of-concept for the load-responsive cellular envelope system, which highlights interesting properties in terms of weight to area ratio, equal to $11 \mathrm{~kg} / \mathrm{m}^{2}$. This is compared with traditional construction skin systems for an intuitive analysis, showing a considerable mass reduction (Table 1). The employed material, High Performance PLA, exhibited ideal characteristics in terms of printability for such geometries, as well as good stiffness and impact resistance.

Table 1: Weight to Area ratio values of typical construction skin systems in comparison with the realized mockup of Load-Responsive Cellular Envelope

\begin{tabular}{|c|c|c|c|c|c|c|}
\hline $\begin{array}{c}\text { CONSTRUCTION } \\
\text { SKIN SYSTEM }\end{array}$ & $\begin{array}{c}\text { Plastered } \\
\text { brickwork }\end{array}$ & Curtain wall & $\begin{array}{c}\text { Balloon frame } \\
\text { construction }\end{array}$ & $\begin{array}{c}\text { Lightweight } \\
\text { Concrete }\end{array}$ & $\begin{array}{c}\text { Precast Concrete } \\
\text { (hollow planks) }\end{array}$ & $\begin{array}{c}\text { Load-responsive } \\
\text { Cellular Envelope }\end{array}$ \\
\hline $\begin{array}{c}\text { WEIGHT TO AREA } \\
\text { RATIO }\left(\mathrm{kg} / \mathrm{m}^{2} \mathrm{~J}\right.\end{array}$ & 339 & 68 & 73 & 268 & 36 & $6 \div 12$ \\
\hline
\end{tabular}

\section{Conclusions}

AM methods have undoubtedly introduced novel materialization processes, where logics of sustainability and efficiency typical of mass-production are no longer applicable. Unprecedented control, precision and freedom of this manufacturing allow the conceptualization of unseen architectural systems. Taking inspiration from the remodelling process of bones, a design methodology which adapts to different shapes and loading conditions is developed. The outcome of this process is an envelope system, which allows the creation of light-permeable load-bearing facades with reduced material usage. This experimental approach challenges current design paradigms of lightweight architecture: complex shapes are neither pre-optimized by shape, nor post-rationalized to meet manufacturing constraints. The system has been successfully tested in a laboratory setup. However, its implementation in operative conditions in buildings would require further developments in terms of material and fabrication equipment. This approach can be easily adapted to the use of metal $3 \mathrm{~d}$ printing, to offer a more robust material option at current time. However, the rapid development of thermos-polymers for $3 d$ printing with increased chemical, mechanical and weather resistance, offers interesting perspectives of application with FDM. Further development of this work will involve the testing in a relevant environment through a full scale architectural demonstrator, where a wider set of evaluative criteria are to be involved. In the long-time perspective, this research offers an alternative approach to the problem of scarcity of resources by shifting the design towards a higher scale of resolution, inferior to $1 \mathrm{~mm}$. At this scale, nature prefers to operate by articulating material into complex formations to minimize the use of material. With this open perspective, complexity in architecture can be considered as a beautiful opportunity.

\section{Acknowledgements}

This work has been developed within the research framework of ACTLAB - Politecnico di Milano. The authors would like to thank to all the researchers and collaborators who have contributed and helped to the research, experiments and developments of this project.

\section{References}

Ashby, M.F. (2005). The properties of foams and lattices, Philosophical Transactions of the Royal Society, No.364, November 2005, pp. 15-30.

Ashby, M.F., Evans, A.G., Fleck, N.A., Hutchinson, J.W., Gibson, L.J. \& Wadley, H. (2000). Metal foams - a design guide, Vol. 1, Butterworth-Heinemann.

Bendsøe, M. P. \& Sigmund, O. (2003). Topology Optimization: Theory, Methods and Application, Berlin, Springer.

Benyus, J. M. (2002). Biomimicry. Innovation inspired by nature, HarperCollins Publishers, NYS.

Gibson, L.J. (2005). Biomechanics of cellular solids, Journal of Biomechanics, No. 38, pp. 377-399.

Gibson, L.J. \& Ashby, M.F. (1997). Cellular Solids: Structure and Properties, 2nd edition, Cambridge University Press, Cambridge.

Hensel, M. (2011). Performance-oriented Architecture and the Spatial and Material Organisation Complex -Rethinking the Definition, Role and Performative Capacity of the Spatial and Material Boundaries of the Built Environment, FORMAkademisk. In Hensel, M. (2013). AD Primer: Performance oriented Architecture, Rethinking Architectural Design and the Built Environment. John Wiley \& Sons, London.

Hooke, R. C. (1665). Micrographia: or Some Physiological Descriptions of Miniature Bodies Made by Magnifying Glasses. London, England: Jo. Martyn, and Ja. Allestree. 
Kim, J.W., Kim, H.S. \& Lee, D.G. (2012). Tensile Strength of Glass Fiber-reinforced Plastic by Fiber Orientation and Fiber Content Variations, International Journal of Modern Physics: Conference Series, Vol. 6, World Scientific, Singapore.

Knippers J., Cremers J., Gabler M. \& Lienhard J. (2011). Construction manual for polymers + membranes. Institut für internationale Architektur-Dokumentation. GmbH \& Co. KG, Munich.

Naboni, R. \& Paoletti, I. (2015). Advanced customization in architectural design and construction, 1st edition, Springer Verlag, 2015.

Naboni, R., Kunic, A. \& Paoletti, I. (2016). Load-responsive skin systems for lightweight architecture. Proceedings of 11th Conference on Advanced Building Skins. Bern, Switzerland. October, 2016.

Turner, J.S. (2012) Evolutionary architecture. Some perspectives from biological design, In AD, Architectural Design: Material Computation, No. 216, March/April 2012, pp. 28-33. 